Comparative Analysis of Land Cover Classification using ML and SVM Classifier for LISS-iv Data

Rahul Neware*1

${ }^{1}$ Department of Computer Science and Engineering, G.H. Raisoni College of Engineering Nagpur, India, Email: neware rahul.ghrcemtechcse@ raisoni.net 


\title{
Comparative analysis of Land cover classification using ML and SVM Classifier for LISS-iv data
}

\begin{abstract}
This paper focuses on the crucial role that remote sensing plays in divining land features. Data that is collected distantly provides information in spectral, spatial, temporal and radiometric domains, with each domain having specific resolution to information collected. Diverse sectors such as hydrology, geology, agriculture, land cover mapping, forestry, urban development and planning, oceanography and others are known to use and rely on information that is gathered remotely from different sensors. In the present study, IRS LISS IV Multi-spectral data is used for land cover mapping. It is known, however, that the task of classifying high-resolution imagery of land cover through manual digitizing consumes time and is way too costly. Therefore, this paper proposes accomplishing classifications by way of enforcing algorithms in computers. These classifications fall in three classes: supervised, unsupervised, and object-based classification. In the case of supervised classification, two approaches are relied upon for land cover classification of high resolution LISS-IV multispectral image. These approaches are: Maximum Likelihood and Support Vector Machine (SVM). Finally, the paper proposes a step-by-step procedure for optical image classification methodology. This paper concludes that in optical data classification, SVM classification gives a better result than ML classification technique.
\end{abstract}

Keywords: Classification, SVM Classifier, ML Classifier, Supervised and Unsupervised Classification, Object-based Classification, Multispectral Data.

\section{Introduction}

Remote sensing has revolutionized the way we gather information. This is because with remote sensing you can garner data or information pertaining to any object from calculations executed at a point away from the object and without the sensor actually coming in contact with it. The earth gives out electromagnetic energy, either self-emitted or reflected/scattered in different bands of wavelengths and the calculation is based on the observation of it. The reflectance/emittance pattern under different spectral/temporal/polarization conditions provides indications or signatures specific to a land feature, which forms the basis for data interpretation (Neware and Khan 2018; Lisani et al. 2016; Schowengerdt 2007). Remotely sensed data provides information in spectral; spatial; temporal; and radiometric domains. Each domain has resolution relevant to the information gathered. Different sensors have various resolutions in these domains (Ran et al. 2017; Cheng et al. 2015; Liu \& Mason 2009; Schott 2007). The finer the resolution, the lesser is the total ground area observed.

This becomes very useful in cartography. In the creation of maps, high-resolution data that is generated by satellite having resolution of 5 to $10 \mathrm{~m}$ in the panchromatic mode is useful, while in the multi-spectral mode 10 to $30 \mathrm{~m}$ is employed to create maps within the scale of 1 : 25,000 and 1: 50,000 (Ajai 2002). Depending on the resolution, the following categories emerge for optical satellite images: Low Resolution, Medium Resolution, High Resolution and Very High-Resolution Satellite Data (Jacobsen 2002). Different types of sensors generate different kinds of data. For instance, multi-spectral sensors like GEOS, Meteosat, NOAA, OCM and MODIS give low resolution optical satellite data with $250 \mathrm{~m}$ - some kms. spatial resolution. And 
multi-spectral sensors like LANDSAT MSS, RESURS-01 (MSU-SK), IRS LISS-I and IRS-1C Wide image Field Sensor (WiFS) generate medium resolution optical satellite data within the range of $50 \mathrm{~m}-250 \mathrm{~m}$ spatial resolution. Panchromatic or multi-spectral sensors or analogue camera systems such as LANDSAT TM, SPOT PAN \& MS, IRS-1C/1D, Resourcesat 1, 2 \& 2A (PAN, LISS III and LISS IV), KFA 1000, MK4, etc. produce high resolution optical satellite data within the scale of $5 \mathrm{~m}-50 \mathrm{~m}$ spatial resolution.

In the present study, IRS LISS IV Multi-spectral data is used, and the details of the sensor are provided in Table 1. (Rsorcesat 2).

Table 1: Specifications of IRS LISS IV sensor

\begin{tabular}{|l|l|}
\hline Sensor & LISS IV \\
\hline Resolution & $5.8 \mathrm{~m}$ (visible and near IR region) \\
\hline Swath & $70 / 23 \mathrm{~km}$ \\
\hline Repetitivity & 5 days \\
\hline Spectral & $0.52-0.59$ microns (B2) \\
Bands & $0.62-0.68$ microns (B3) \\
& $0.77-0.86$ microns (B4) \\
\hline Radiometry & 10 -bit \\
\hline
\end{tabular}

Preceding researchers have established that that using different classifiers may lead to different outcomes of classification. Consequently, to confirm the efficacy of different classifiers a plethora of research has been done. (Thilagavati 2015; Zewdie 2015; Sexton 2013; Cheng et al. 2013; Longbotham et al. 2012; Yi \& Newsam 2011; Prakasam 2010; Yang \& Newsam 2010; Xin, Liangpei \& Le 2009).

However, these studies had been mainly carried out using pixel-based approaches. There are some basic principles for the selection of training samples for pixel-based classification (Van Niel et al. 2005; Foody et al. 2006).

Based on the information required in land cover classification, six classes were identified: agriculture land, water body, settlement and scrub areas in forest land entwined with agricultural land. In this, the sample collection is the most time-consuming and a critical process in land cover classification efforts. This research study randomly chose samples from recognized regions through the use of ENVI version 5.0 software and its 'region of interest' (RoI) tools, together with the help of image characteristics and ground know-how. Those homogeneous sample areas had been easily visually recognized on the LISS-IV image and Bhuvan imagery. It was found that the sample pixels are distributed evenly and uniformly, thus effectively representing the whole study area. In their study (Jia et al. 2014), they randomly chose, as training samples, 50\% of the sample pixels, and used the rest to gauge the accuracy of classification.

\section{Classification Techniques of Land Use}

It is worth noting that different sensors using various platforms and giving a wide range of spectral, spatiotemporal and radiometric resolutions have served to make available data that is remotely sensed. Consequently, numerous sectors including geology, land cover mapping, oceanography, hydrology, urban development and planning, forestry and agriculture have made extensive use of this technology (Melesse et al. 2007; Acharya et al. 2016).

Land cover information that can be gleaned from satellite images are the spectral and spatial attributes of individual cover types. There are some differences between coarse and fine 
resolution data, mainly in relative importance of these two kinds of attributes. Because of the reduced resolution, the spectral dimension is the most important source of cover type information in coarse resolution images. For fine resolution data, the relative importance of spatial dimension is higher, although the spectral content still dominates in most cases (RSA Document).

Land cover classification of high-resolution imagery can be performed via manual digitizing on a computer display screen. As manual digitizing takes a lot of time and is costly, the classification is generally accomplished by way of enforcing algorithms in computers. Various classification techniques that have been advanced in latest decades can be classified into three huge classes: supervised, unsupervised, and object-based classification.

\subsection{Supervised Classification}

Supervised classification is the type of machine learning in which training input is given, and then according to training inputs classification is applied. Supervised classification is the most used classification algorithm in remote sensing. After applying this technique, output is classified image according to each class called training. In supervised classification the user has to select region of interest which acts as classifier, and pixels of whole image are classified according to region of interest. In remote sensing, while performing supervised classification, maximum likelihood and Spectral mapping classification algorithms are mostly used. (Dingwen et al. 2015; Munoz-Mari \& Camps-Valls 2012; Atkinson \& Lewis 2000).

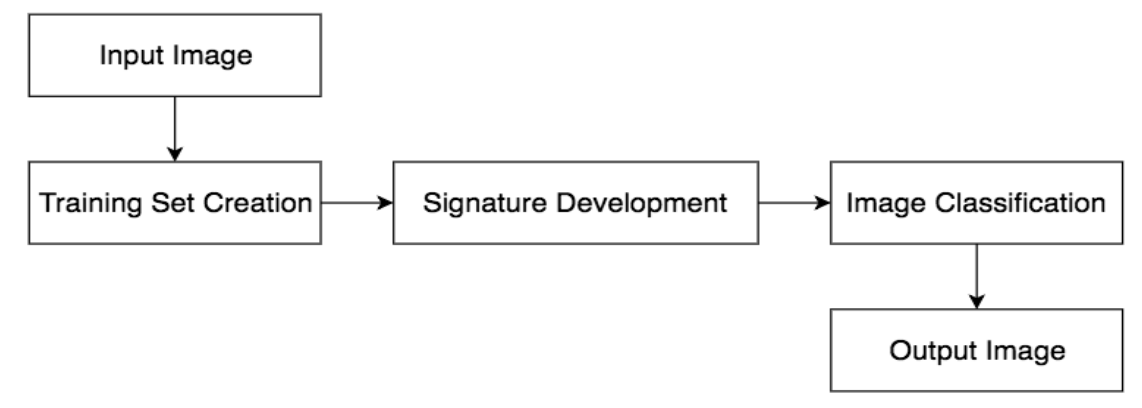

Figure 1. Supervised Classification Technique

\subsection{Unsupervised Classification}

Unsupervised classification denotes that the analyst, during the classifying stage, has no advanced knowledge. It is normally carried out purely based on the naturally occurring spectrum distribution of features of ground objects in the images that are sensed remotely. Unsupervised classification chiefly relies on cluster analysis. Clustering is defined as segmenting pixels into numerous classes with the aim of grouping pixels having similar characteristics. The aim to make sure that between pixels in the same class the distance is as little as possible, and between pixels of different classes, the distance is as large as possible. Unsupervised classification centres around the choice of the initial class parameters and adjustments in its iteration. The task of giving land cover labels to individual spectral clusters is accomplished later, on the basis of ground data culled from the areas indicated by the resulting clusters (Yuan, Wan \& Wang 2016; Cheriyadat 2014). 


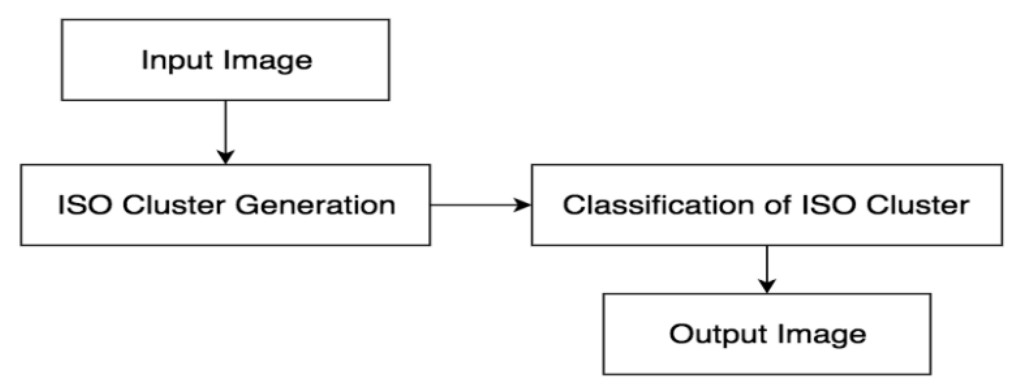

Figure 2. Unsupervised Classification Technique

\subsection{Object-Based Classification}

Object-oriented classification is the alternative for pixel-based classification. Object-oriented classification is specially used for high resolution imagery. In object-oriented classification a satellite image is mainly segmented into objects. Hence, it is called object-oriented classification. In object-oriented classification a knowledge base is created for the segmented object like, agency, length, shape and size etc. The object-oriented classification gives very accurate classified results of high-resolution satellite images (Blaschke 2010; Li, Gu, Han, \& Yang 2010).

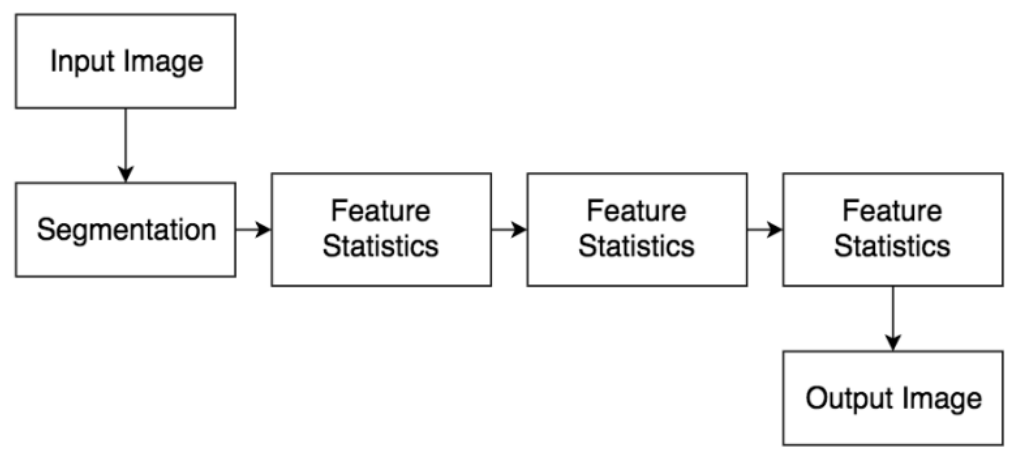

Figure 3. Object-Based Classification Technique

\section{Classification Techniques Used}

This paper presents the use of two classification algorithms: Maximum likelihood and Support Vector Machine algorithm, which are as follows:

\subsection{Maximum Likelihood Classifier}

The most widely used classification in remote sensing is the maximum likelihood classifier. This is because it uses a simple premise, conjecturing on the probability or chance of a pixel fitting into a specific category or class. And its fundamental equation presumes that for all the categories or classes these probabilities are same, and the bands that are put in are normally distributed. However, the probabilities are not equal for all classes. For instance, one can specify weight factor for a particular class. This variation of the maximum likelihood decision rule is known as the Bayesian decision rule (Sisodia, Tiwari \& Kumar 2014; Hester et al. 2008; Bruzzone \& Prieto 2001; Paola \& Schowengerdt 1995; Hord 1982).

The equation for the maximum likelihood/ Bayesian classifiers is as fallows. 


$$
L_{k}=P\left(\frac{k}{X}\right)=P(K)^{*} P(X / k) / \sum P(i)^{*} P(X / i)
$$

WhereP(k): prior probability of class $\mathrm{k}$

$\mathrm{P}(\mathrm{X} / \mathrm{k})$ : conditional probability to observe $\mathrm{X}$ from class $\mathrm{k}$, or probability density function Usually $\mathrm{P}(\mathrm{k})$ are assumed to be equal to each other and $\sum \mathrm{P}(\mathrm{i})^{*} \mathrm{P}\left(\frac{\mathrm{X}}{\mathrm{i}}\right)$ is also common to all classes. Therefore $\mathrm{L}_{\mathrm{k}}$ depends on $\mathrm{P}(\mathrm{X} / \mathrm{k})$ or the probability density function.

\subsection{Support Vector Machine Classifier}

Support Vector Machine (SVM), essentially, is an algorithm of supervised machine learning. Although this algorithm can be employed for classification as well as for challenges of regression, it is primarily used to solve issues of classification. Support vector machines (SVMs, also support vector networks) (Cortes \& Vapnik 1995) double up as supervised learning entities with linked algorithms of learning, which interpret data that is utilized for classification and for analyzing regression. When presented with a bunch of training examples, the algorithm marks each example placing it either one or the other of twin categories. Thus an SVM training algorithm constructs a facsimile .which allocates new examples to one or the other category, thus creating a classifier that is non-probabilistic binary linear.

As a classification approach SVM is extracted from the theory of statistical learning. What it does is to sunder the classes with a decision surface that widens the boundaries between the classes. Here the surface is frequently billed as the optimal hyperplane, and points of data that are nearest to the hyperplane are billed as support vectors which, in turn, are the crucial aspects of the training set. Particularly, from data that is noisy and complex, excellent classification outcomes are given by SVM.

Interestingly, by utilizing non-linear kernels SVM can be adapted to create a non-linear classifier. Also, because SVM is essentially a pure binary classifier, it can double up as a multiclass classifier by stringing together many binary SVM classifiers, basically producing, for each separate pair of classes, a binary classifier. For multi-class classification, SVM employs a strategy to classify pairs. The output of SVM classification translates into the decision parameters or values of each pixel for each class which, in turn, are utilized to gauge the probability. And when each probability falls in the margin of 0 to 1 , these probability values are deemed as "true" probability and the total of these values for each pixel amounts to 1 . By choosing the highest probability classification is accomplished. (Tuia et. al 2014; Moustakidis et al. 2012).

\section{Methodology}

In this study, first of all, the area pertaining to the study area was geo-referenced to address geometric distortions with the help Ground Control Points (GCPs). Using information gathered from field visit and knowledge of experts, from the high resolution image samples for training and validation were secured. Figure 4 shows the entire process this study adopted. The step-bystep procedure of the optical image classification methodology is as follows:

Step 1: Obtain the LISS-IV optical image of the study area.

Step 2: Perform rectification on the obtained LISS-IV image of the study area (Roy 2010; Ju 2012).

Step 3: Apply the supervised classification of various algorithms on the rectified image of LISS-IV image. 
Step 4: Obtain the classified image as output and graphical representation of each band. The broad methodology followed for the supervised classification is depicted in Figure 4. To compare the outcomes of correctness, on the original dataset ML and SVM, two of the most widely employed classifiers, were applied.

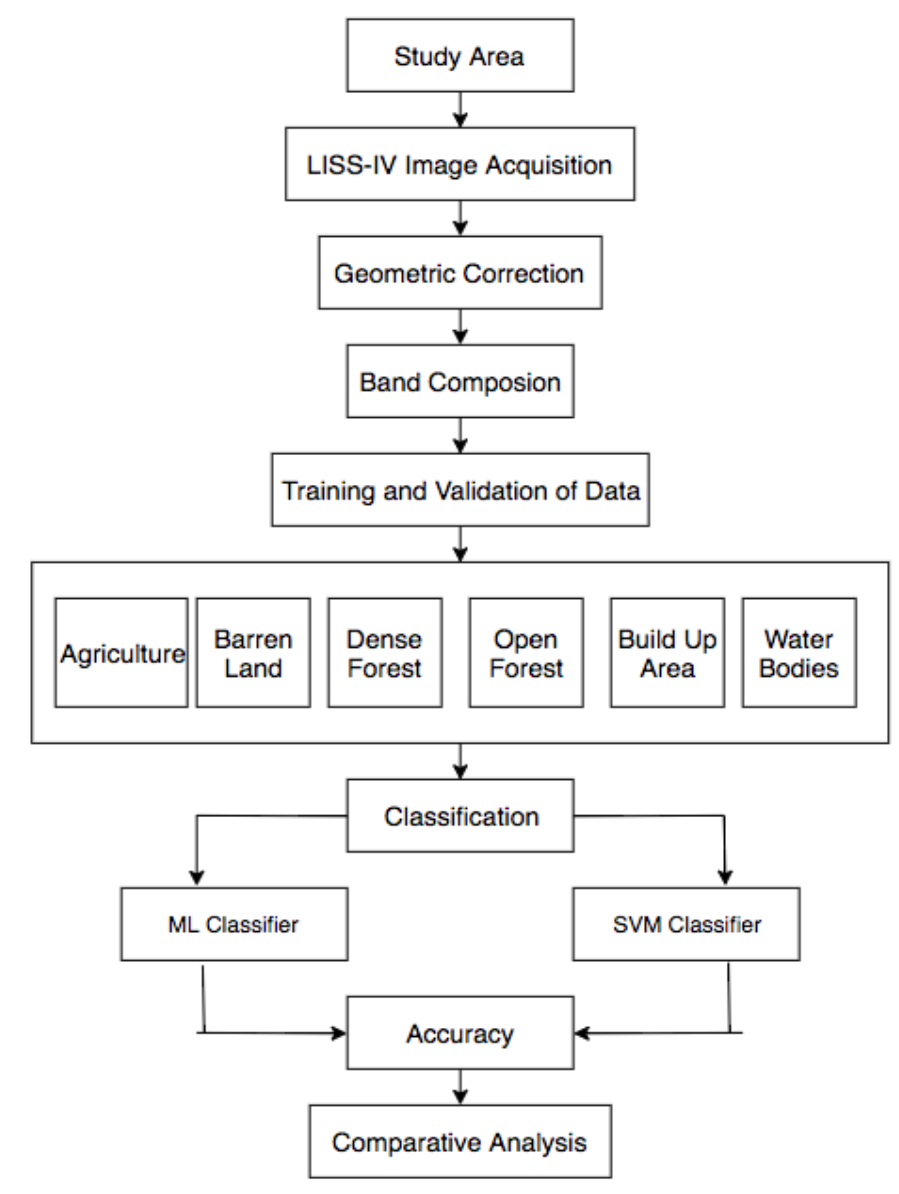

Figure 4. Methodology for the classification LISS-IV images.

\subsection{Working of Maximum Likelihood}

The maximum likelihood technique has been used to classify high resolution LISS IV imagery. The ground truth data collected during field data collection phase has been incorporated into the classification system by drawing Region of Interest (ROI) polygons in ENVI software. The satellite image overlaid with corresponding ROIs is presented in Figure 5. The standard deviation and mean values of the classes are presented in Figure 6. 


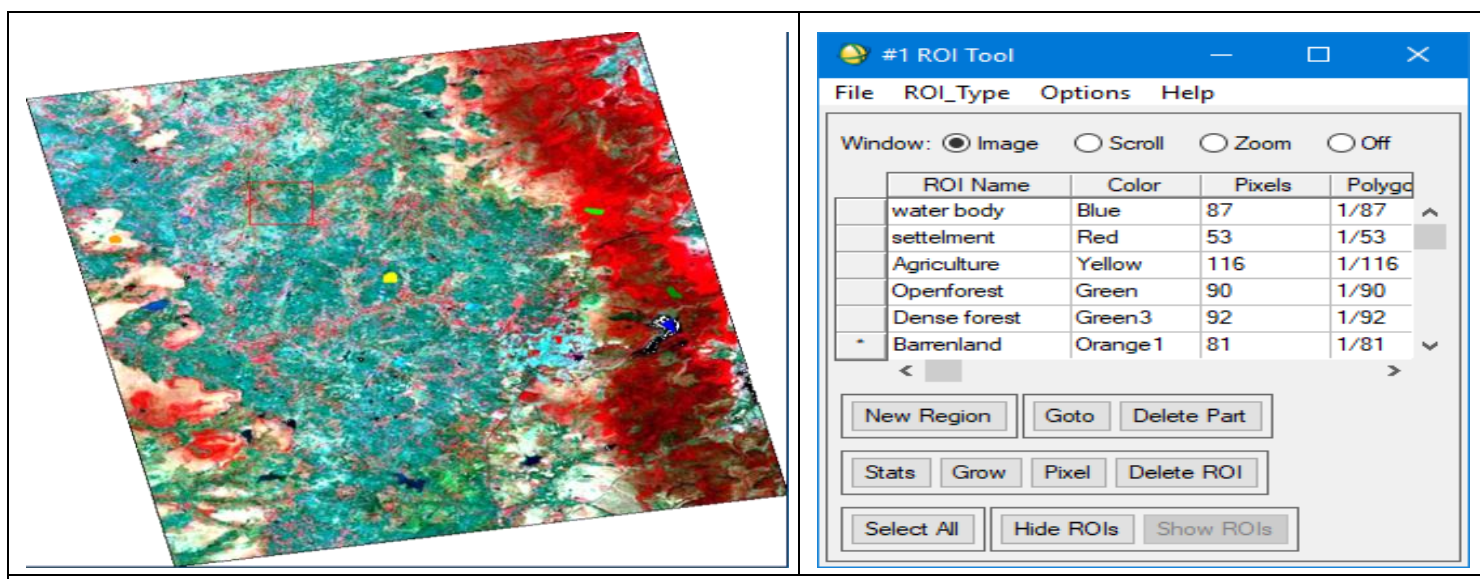

Figure 5. LISS IV imagery with ROIs depicting different land cover classes

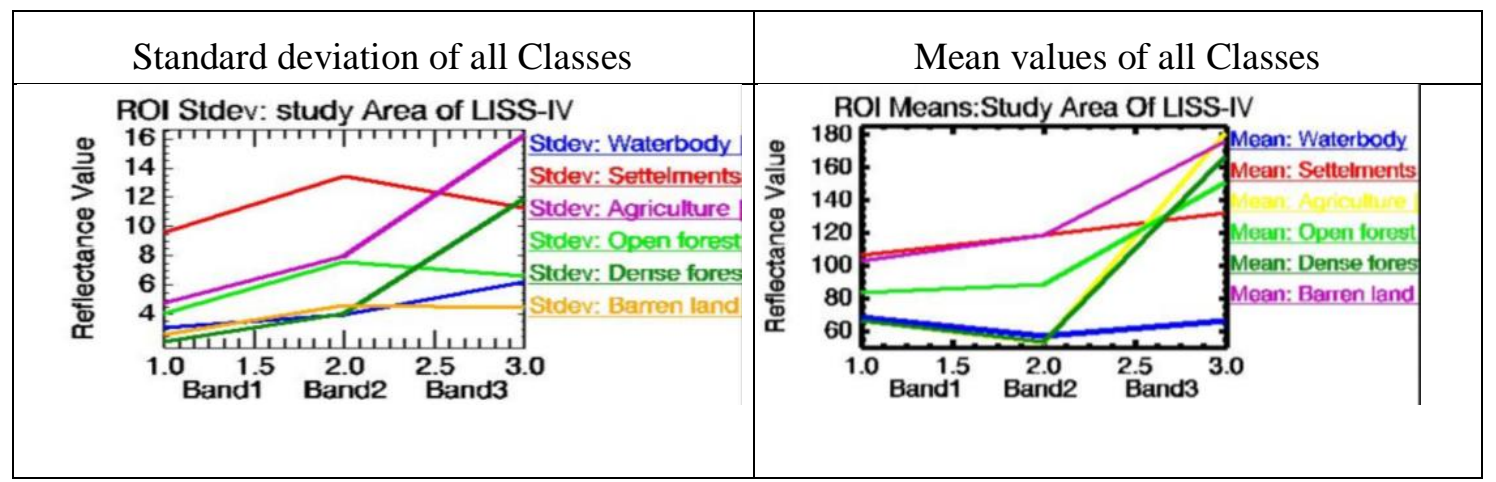

Figure 6. Standard deviation and mean values of selected classes

The accuracy assessment is carried out with the help of confusion matrix and kappa accuracies and is presented in Tables 2 and 3 respectively.

Table 2. Confusion Matrix for the Maximum likelihood classification

\begin{tabular}{cllllll}
\hline $\begin{array}{c}\text { Classification of } \\
\text { LISS-IV Image }\end{array}$ & \multicolumn{7}{c}{ ML Classifier } \\
\hline Land cover classes & 1 & 2 & 3 & 4 & 5 & 6 \\
Water body & 90 & 3 & 0 & 0 & 0 & 7 \\
Settlements & 0 & 66 & 24 & 10 & 0 & 0 \\
Agriculture & 8 & 2 & 61 & 10 & 9 & 10 \\
Open forest & 0 & 4 & 10 & 80 & 0 & 6 \\
Dense forest & 0 & 15 & 5 & 0 & 80 & 0 \\
Barren land & 2 & 10 & 0 & 0 & 11 & 77 \\
\hline
\end{tabular}

Table 3. Overall accuracy and kappa coefficient for the ML Classified output 


\begin{tabular}{lcc}
\hline LISS-IV Image & Overall Accuracy & Kappa Coefficient \\
\hline MLC & $75.7 \%$ & $0.70 \%$ \\
\hline
\end{tabular}

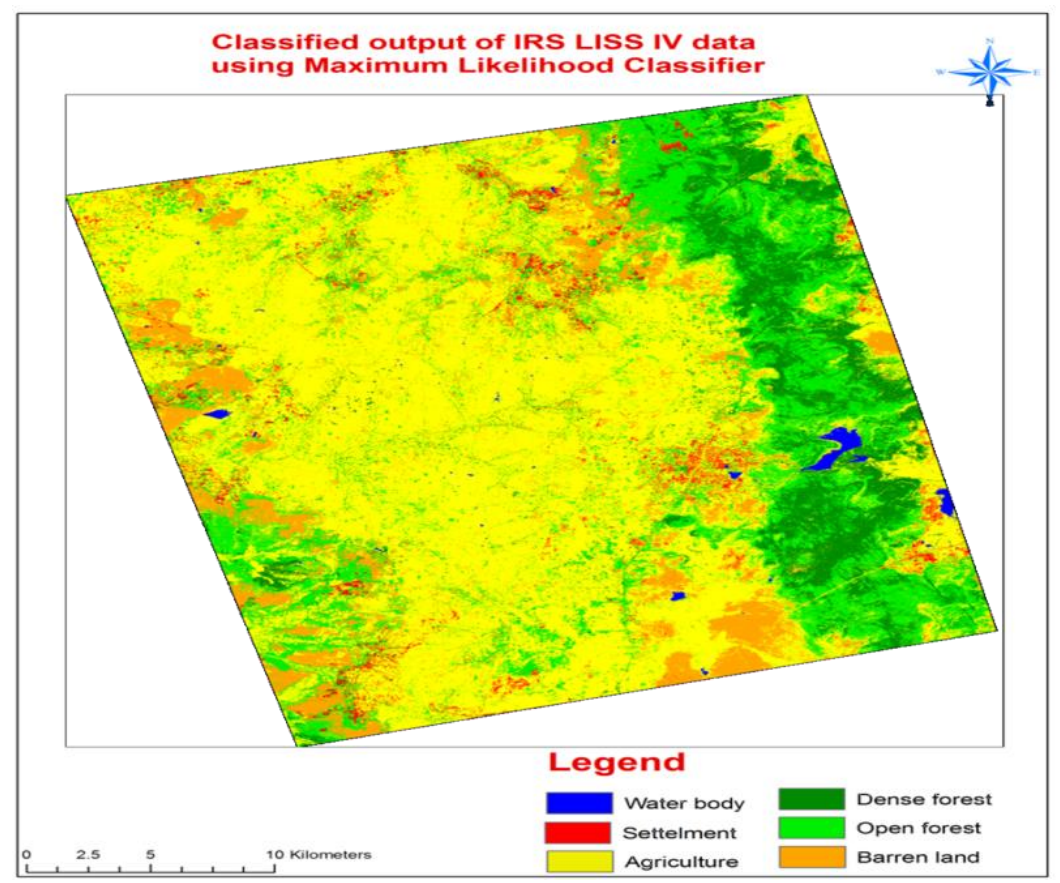

Figure 7. Output of ML Classification

\subsection{Working of SVM Classifier}

The SVM technique has been used to classify high resolution LISS IV imagery. The ground truth data collected during field data collection phase has been incorporated into the classification system by drawing Region of Interest (ROI) polygons in ENVI software. In fact, the same ground truth data that was used for MXL classified has also been used in SVM classification. The satellite image overlaid with corresponding RoIs is presented in figure 8 . The standard deviation and mean values of the classes are presented in figure 6 .

The accuracy assessment is carried out with the help of confusion matrix and kappa accuracies and is presented in tables 4 and 5 respectively.

Table 4. Confusion Matrix for the SVM classification

\begin{tabular}{cllllll}
\hline $\begin{array}{c}\text { Classification of } \\
\text { LISS-IV Image }\end{array}$ & & \multicolumn{5}{c}{ SVM } \\
\hline Land cover classes & 1 & 2 & 3 & 4 & 5 & 6 \\
Water body & 95 & 4 & 0 & 0 & 1 & 0 \\
Settlements & 0 & 52 & 25 & 5 & 8 & 10 \\
Agriculture & 5 & 12 & 65 & 10 & 1 & 7 \\
Open forest & 0 & 8 & 4 & 85 & 2 & 1 \\
Dense forest & 0 & 10 & 0 & 0 & 88 & 2 \\
Barren land & 0 & 14 & 6 & 0 & 0 & 80 \\
\hline
\end{tabular}


Table 5. Overall accuracy and kappa coefficient for the SVM Classified output

\begin{tabular}{lcc} 
LISS-IV Image & Overall Accuracy & Kappa Coefficient \\
\hline SVM & $77.5 \%$ & $0.75 \%$ \\
\hline
\end{tabular}

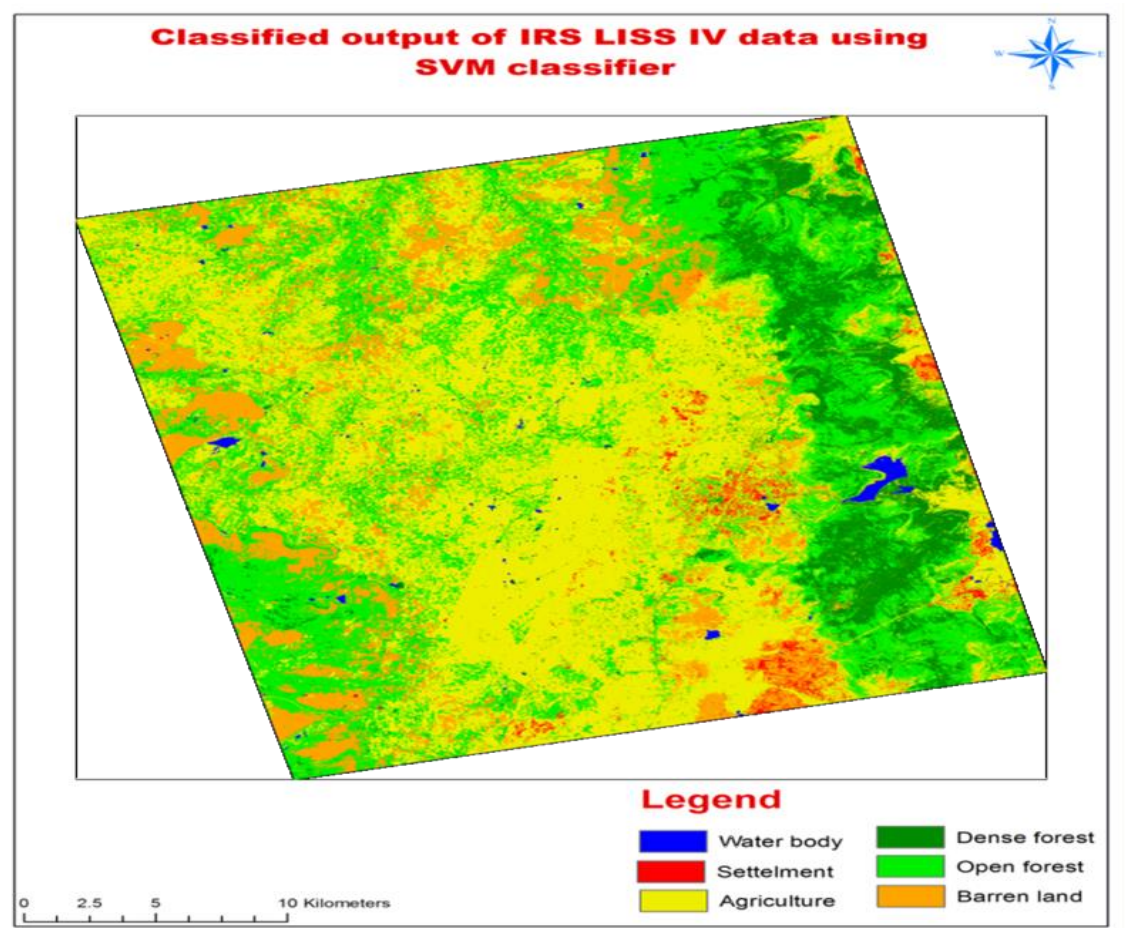

Figure 8. Output of SVM Classification

\section{Result and Conclusion}

In optical data classification, too, ML and SVM classification techniques were used. A comparative analysis of MXL and SVM classification reveals that SVM classification gives a better result than MXL with an overall accuracy of $77.5 \%$ and kappa coefficient of 0.75 , whereas with MXL it is $75.7 \%$ and 0.70 respectively. SVM shows better results for all classes except for settlement in which MXL shows better results.

Table 6. Comparative Analysis of Different land cover classification results

\begin{tabular}{cllllll|llllll}
\hline $\begin{array}{c}\text { Classification of } \\
\text { LISS-IV Image }\end{array}$ & \multicolumn{4}{c|}{ SVM } & \multicolumn{7}{c}{ ML } \\
\hline Land cover classes & 1 & 2 & 3 & 4 & 5 & 6 & 1 & 2 & 3 & 4 & 5 & 6 \\
Water body & 95 & 4 & 0 & 0 & 1 & 0 & 90 & 3 & 0 & 0 & 0 & 7 \\
Settlements & 0 & 52 & 25 & 5 & 8 & 10 & 0 & 66 & 24 & 10 & 0 & 0 \\
Agriculture & 5 & 12 & 65 & 10 & 1 & 7 & 8 & 2 & 61 & 10 & 9 & 10 \\
Open forest & 0 & 8 & 4 & 85 & 2 & 1 & 0 & 4 & 10 & 80 & 0 & 6 \\
Dense forest & 0 & 10 & 0 & 0 & 88 & 2 & 0 & 15 & 5 & 0 & 80 & 0 \\
Barren land & 0 & 14 & 6 & 0 & 0 & 80 & 2 & 10 & 0 & 0 & 11 & 77 \\
\hline
\end{tabular}

Table 7. Comparison of overall accuracy and kappa coefficient 


\begin{tabular}{ccc}
\hline LISS-IV Image & Overall Accuracy & Kappa Coefficient \\
\hline SVM & $77.5 \%$ & 0.75 \\
MLH & $75.7 \%$ & 0.70 \\
\hline
\end{tabular}

\section{References}

Neware R., Khan A., (2018), "IDENTIFICATION OF AGRICULTURE AREAS IN SATELLITE IMAGES USING SUPERVISED CLASSIFICATION TECHNIQUE", INTERNATIONAL JOURNAL OF CREATIVE RESEARCH THOUGHTS (IJCRT), 6(2), pp.682-688.

Lisani, J. L.; Michel, J.; Morel, J.M.; Petro, A.B.; Sbert, C.(2016). An inquiry on contrast enhancement methods for satellite images. IEEE Trans. Geosci. Remote Sens. Vol. 54, no.12, pp. 7044-7054, 2016.

Schowengerdt, Robert A. (2007). Remote sensing: models and methods for image processing (3rd ed.). Academic Press. p. 2. ISBN 978-0-12-369407-2.

Ran, L., Zhang, Y., Wei, W., \& Zhang, Q. (2017). A Hyperspectral Image Classification Framework with Spatial Pixel Pair Features. Sensors, 17(10), 2421. Doi: $10.3390 / \mathrm{s} 17102421$.

Cheng, G., Han, J., Guo, L., Liu, Z., Bu, S., \& Ren, J. (2015). Effective and Efficient Midlevel Visual Elements-Oriented Land-Use Classification Using VHR Remote Sensing Images. IEEE Transactions on Geoscience and Remote Sensing, 53(8), 4238-4249. Doi: $10.1109 /$ tgrs.2015.2393857.

Liu, Jian Guo \& Mason, Philippa J. (2009). Essential Image Processing for GIS and Remote Sensing. Wiley-Blackwell. p. 4. ISBN 978-0-470-51032-2.

Schott, John Robert (2007). Remote sensing: the image chain approach (2nd ed.). Oxford University Press. p. 1. ISBN 978-0-19-517817-3.

Ajai. (2002). Mapping for Micro-Level Planning: The Issues \& Challenges. Proceedings, INCA Conference, Indian Cartographer.

Jacobsen, K. (2002). Comparison of high-resolution mapping from space. Indian Cartographer, 22, 106-119.

Thilagavati, N., Subramani, T., Suresh, M. (2015). Land use/land cover change detection analysis in Salem Chalk Hills, South India using remote sensing and GIS. Disaster Adv. 44-52.

Zewdie, W., \& Csaplovies, E. (2015). Remote Sensing based multi-temporal land cover classification and change detection in northwestern Ethiopia. European Journal of Remote Sensing, 48(1), 121-139. Doi:10.5721/eujrs20154808

Sexton, J. O., Urban, D. L., Donohue, M. J., \& Song, C. (2013). Long-term land cover dynamics by multi-temporal classification across the Landsat-5 record. Remote Sensing of Environment, 128, 246-258. Doi: 10.1016/j.rse.2012.10.010.

Cheng, G., Guo, L., Zhao, T., Han, J., Li, H., and Fang, J., (2013). Automatic landslide detection from remote-sensing imagery using a scene classification method based on BoVW and 
pLSA. International Journal of Remote Sensing, $34 \quad$ (1), 45-59. Doi:10.1080/01431161.2012.705443.

Longbotham, N., Chaapel, C., Bleiler, L., Padwick, C., Emery, W. J., \& Pacifici, F. (2012). Very High Resolution Multiangle Urban Classification Analysis. IEEE Transactions on Geoscience and Remote Sensing, 50(4), 1155-1170. Doi:10.1109/tgrs.2011.2165548.

Yi Yang, \& Newsam, S. (2011). Spatial pyramid co-occurrence for image classification. 2011 International Conference on Computer Vision. doi:10.1109/iccv.2011.6126403.

Prakasam, C. (2010). Land use land cover change detection through remote sensing approach: a case study of Kodaikaikanal taluk, Tamil Nadu. Int J Geomatics Geosi. Pp:150-158.

Yang, Y., \& Newsam, S. (2010). Bag-of-visual-words and spatial extensions for land-use classification. Proceedings of the 18th SIGSPATIAL International Conference on Advances in Geographic Information Systems - GIS ’10. Doi:10.1145/1869790.1869829.

Xin Huang, Liangpei Zhang, \& Le Wang. (2009). Evaluation of Morphological Texture Features for Mangrove Forest Mapping and Species Discrimination Using Multispectral IKONOS Imagery. IEEE Geoscience and Remote Sensing Letters, 6(3), 393-397. Doi:10.1109/lgrs.2009.2014398.

Van Niel, T.G. and McVicar, T.R. (2004) Determining Temporal Windows for Crop Discrimination with Remote Sensing: A Case Study in South-Eastern Australia. Computers and Electronics in Agriculture, 45, 91-108. http://dx.doi.org/10.1016/j.compag.2004.06.003

Foody, G. M., Mathur, A., Sanchez-Hernandez, C., \& Boyd, D. S. (2006). Training set size requirements for the classification of a specific class. Remote Sensing of Environment, 104(1), 1-14. Doi:10.1016/j.rse.2006.03.004

Kun Jia, Xiangqin Wei, Xingfa Gu, Yunjun Yao, Xianhong Xie \& Bin Li (2014) Land cover classification using Landsat 8 Operational Land Imager data in Beijing, China, Geocarto International, 29:8, 941-951, DOI: 10.1080/10106049.2014.894586

Jia, K., Liang, S., Zhang, N., Wei, X., Gu, X., Zhao, X., Xie, X. (2014). Land cover classification of finer resolution remote sensing data integrating temporal features from time series coarser resolution data. ISPRS Journal of Photogrammetry and Remote Sensing, 93, 4955. Doi: 10.1016/j.isprsjprs.2014.04.004

Melesse, A. M., Weng, Q., Thenkabail, P. S., \& Senay, G. B. (2007). Remote sensing sensors and applications in environmental resources mapping and modeling. Sensors, 7(12), 32093241. Doi: 10.3390/s7123209.

Dowman, I. (2001). High resolution satellite data: Status and Issues. High Resolution Mapping from Space.

Acharya, T., Yang, I., \& Lee, D. (2016). Land Cover Classification Using a KOMPSAT-3A Multi-Spectral Satellite Image. Applied Sciences, 6(11), 371. Doi:10.3390/app6110371.

Munoz-Mari, J., Tuia, D., \& Camps-Valls, G. (2012). Semisupervised Classification of Remote Sensing Images With Active Queries. IEEE Transactions on Geoscience and Remote Sensing, 50(10), 3751-3763. Doi:10.1109/tgrs.2012.2185504. -Supervised

Dingwen Zhang, Junwei Han, Gong Cheng, Zhenbao Liu, Shuhui Bu, \& Lei Guo. (2015). Weakly Supervised Learning for Target Detection in Remote Sensing Images. IEEE Geoscience 
and Remote Sensing Letters, 12(4), 701-705. Doi:10.1109/lgrs.2014.2358994. Supervised

Atkinson, P. ., \& Lewis, P. (2000). Geostatistical classification for remote sensing: an introduction. Computers \& Geosciences, 26(4), 361-371. Doi:10.1016/s00983004(99)00117-x.

Yuan, Y., Wan, J., \& Wang, Q. (2016). Congested scene classification via efficient unsupervised feature learning and density estimation. Pattern Recognition, 56, 159-169. Doi:10.1016/j.patcog.2016.03.020.

Cheriyadat, A. M. (2014). Unsupervised Feature Learning for Aerial Scene Classification. IEEE Transactions on Geoscience and Remote Sensing, 52(1), 439-451. doi:10.1109/tgrs.2013.2241444.

Blaschke, T. (2010). Object based image analysis for remote sensing. ISPRS Journal of Photogrammetry and Remote Sensing, 65(1), 2-16. Doi:10.1016/j.isprsjprs.2009.06.004

Li, H., Gu, H., Han, Y., \& Yang, J. (2010). Object-oriented classification of high-resolution remote sensing imagery based on an improved colour structure code and a support vector machine. International Journal of Remote Sensing, 31(6), 1453-1470. Doi:10.1080/01431160903475266.

Sisodia, P. S., Tiwari, V., \& Kumar, A. (2014). Analysis of Supervised Maximum Likelihood Classification for remote sensing image. International Conference on Recent Advances and Innovations in Engineering (ICRAIE-2014). Doi:10.1109/icraie.2014.6909319.

Hester, D. B., Cakir, H. I., Nelson, S. A. C., \& Khorram, S. (2008). Per-pixel Classification of High Spatial Resolution Satellite Imagery for Urban Land-cover Mapping. Photogrammetric Engineering \& Remote Sensing, 74(4), 463-471. Doi:10.14358/pers.74.4.463

Bruzzone, L., \& Prieto, D. F. (2001). Unsupervised retraining of a maximum likelihood classifier for the analysis of multitemporal remote sensing images. IEEE Transactions on Geoscience and Remote Sensing, 39(2), 456-460. Doi:10.1109/36.905255. -ML

Paola, J. D., \& Schowengerdt, R. A. (1995). A detailed comparison of backpropagation neural network and maximum-likelihood classifiers for urban land use classification. IEEE Transactions on Geoscience and Remote Sensing, 33(4), 981-996. Doi:10.1109/36.406684.-ML

Hord R. M. (1982). Digital Image Processing of Remotely-Sensed Data. Elsevier, ISBN$0323162355,9780323162357$.

Cortes, C., \& Vapnik, V. (1995). Support-vector networks. Machine Learning, 20(3), 273-297. Doi:10.1007/bf00994018

Tuia, D., Volpi, M., Dalla Mura, M., Rakotomamonjy, A., \& Flamary, R. (2014). Automatic Feature Learning for Spatio-Spectral Image Classification with Sparse SVM. IEEE Transactions on Geoscience and Remote Sensing, 52(10), 6062-6074. Doi: 10.1109/tgrs.2013.2294724-SVM

Moustakidis, S., Mallinis, G., Koutsias, N., Theocharis, J. B., \& Petridis, V. (2012). SVM-Based Fuzzy Decision Trees for Classification of High Spatial Resolution Remote Sensing 
Images. IEEE Transactions on Geoscience and Remote Sensing, 50(1), 149-169. Doi: 10.1109/tgrs.2011.2159726-SVM

Roy D, Junchang J, Kristi K, et al. (2010). Web-enabled Landsat Data (WELD): Landsat ETM+ composited mosaics of the conterminous United States. Remote Sens Environ.114:3549.

Ju J, David P, Eric V, Jeffrey M, Valeriy K. (2012). Continental-scale validation of MODIS-based and LEDAPS Landsat ETM+ atmospheric correction methods. Remote Sens Environ. 122:175-184. 